\title{
Tecnologias de acesso livre para georreferenciamento e análise de sistemas e redes de atenção aos usuários de drogas
}

\author{
Open access technologies to georeferencing and care system and \\ network analysis for drug users
}

\section{Acceso abierto tecnologías para georreferenciación y análisis de sistemas y redes de atención a los usuarios de drogas}

\author{
Pedro Henrique Antunes da Costa | phantunes.costa@gmail.com \\ Universidade Federal de Juiz de Fora, Programa de Pós-Graduação em Psicologia. Juiz de Fora, Brasil. \\ Leonardo Fernandes Martins | leomartinsjf@gmail.com \\ Universidade Federal de Juiz de Fora, Programa de Pós-Graduação em Psicologia. Juiz de Fora, Brasil. \\ Telmo Mota Ronzani | tm.ronzani@gmail.com \\ Universidade Federal de Juiz de Fora, Programa de Pós-Graduação em Psicologia. Juiz de Fora, Brasil. \\ Fernando Antonio Basile Colugnati | fcolugnati@gmail.com \\ Universidade Federal de Juiz de Fora, Programa de Pós-Graduação em Psicologia. Juiz de Fora, Brasil.
}

\section{Resumo}

Este artigo tem como objetivo apresentar um método de georreferenciamento e análise de sistemas de cuidado aos usuários de drogas baseado em tecnologias e processos de acesso livre que podem ser utilizados em diferentes cenários. O método é constituído de três etapas: 1) definição conceitual dos sistemas e das redes de atenção aos usuários de drogas; 2) levantamento dos serviços e o seu mapeamento por georreferenciamento; e 3) caracterização dos relacionamentos entre os serviços da rede a partir da análise de redes sociais (ARS). De modo a demonstrar suas potencialidades e revelar outras possibilidades, o presente método foi utilizado em um município brasileiro de médio/grande porte. Foram identificados e georreferenciados 187 serviços, e suas interações caracterizadas pela ARS. Concluiu-se que merecem destaque três potencialidades do método proposto: 1) o caráter ampliado proporcionado pela abordagem de redes; 2) o baixo custo e a utilização de ferramentas inovadoras e acessíveis; e 3) auxílio para a visualização e o gerenciamento da rede.

Palavras-chave: atenção à saúde; transtornos relacionados ao uso de substâncias; saúde mental; redes sociais; mapeamento geográfico. 


\begin{abstract}
This article aims to present a method of georeferencing and analysis of care systems for drug users based on free access technologies and processes that can be used in different scenarios. The method is composed of three steps: 1) conceptual definition of care systems and networks for drug users; 2) survey of services and their mapping by georeferencing; and 3) characterization of the relationships between the network services based on social network analysis (SNA). In order to demonstrate its potentialities and to reveal other possibilities, the present method was used in a Brazilian medium/large municipality. One hundred and eighty seven services were identified and georeferencied, and their interactions were characterized by the SNA. It was found that is important to underline the following potentialities of the method proposed: 1) the extended character provided for a network approach; 2) the low cost and the use of innovative and accessible tools; and 3) its support to someone visualizing and managing the network.
\end{abstract}

Keywords: health care; substance-related disorders; mental health, social network analysis; geographic mapping.

\title{
Resumen
}

Este artículo tiene el objetivo de presentar un método de georreferenciación y análisis de sistemas de asistencia a los usuarios de drogas basado en tecnologías y procesos de libre acceso que pueden ser utilizados en diferentes escenarios. El método es constituido por tres etapas: 1) definición conceptual de los sistemas y redes de atención a los consumidores de drogas; 2) encuesta de servicios y su representación espacial por medio de la georreferenciación; y 3) caracterización de las relaciones entre los servicios de red a partir del análisis de redes sociales (ARS). Con el fin de demostrar su potencial y revelar otras posibilidades, se utilizó este método en un municipio brasileño de tamaño medio/grande. Ciento y ochenta y siete servicios fueron identificados y georreferenciados, con sus interacciones caracterizadas por la ARS. Merecen ser resaltados los siguientes beneficios potenciales: 1) el carácter ampliado proporcionado por el enfoque de redes; 2) el bajo coste y el uso de herramientas innovadoras y disponibles; 3) ayuda para la visualización y gestión de la red.

Palabras clave: atención a la salud; transtornos relacionados con drogas; salud mental; red social; posicionamiento geográfico.

interpretação dos dados e elaboração do artigo.

Declaração de conflito de interesses: não há.

Fontes de financiamento: Fapemig; Capes; CNPq.

Considerações éticas: não há.

Agradecimento/Contribuições adicionais: não há.

Histórico do artigo: Submetido: 24.mar.2017 | Aceito: 22.nov.2017 | Publicado: 29.dez.2017

Apresentação anterior: não houve.

Licença CC BY-NC atribuição não comercial. Com essa licença é permitido acessar, baixar (download), copiar, imprimir, compartilhar, reutilizar e distribuir os artigos, desde que para uso não comercial e com a citação da fonte, conferindo os devidos créditos de autoria e menção à Reciis. Nesses casos, nenhuma permissão é necessária por parte dos autores ou dos editores. 


\section{Introdução}

Devido às carências nos sistemas de saúde em todo o mundo, uma avaliação ganha importância, devendo ser continuamente discutida e repensada, de modo a considerar as constantes mudanças societárias, políticas, bem como evoluções teóricas e metodológicas. A própria viabilidade de uso das avaliações relaciona-se com a rapidez dos procedimentos avaliativos e a acessibilidade das ferramentas de coleta e análise dos dados.

A partir do desenvolvimento de tecnologias na área de e-health (novas tecnologias da informação e comunicação aplicadas à área da saúde) é possível a construção de métodos de avaliação rápidos, bem estruturados e de baixo custo ${ }^{1}$. Duas tecnologias são fundamentais para esse processo: 1) a primeira é o uso de cloud computing, através do emprego de ferramentas disponíveis na internet e de acesso livre e gratuito, que possibilitam diminuir o tempo para o processamento, armazenamento, organização e análise ${ }^{2} ; 2$ ) a segunda está relacionada com o emprego de dispositivos móveis como smartphones e/ou tablets associados ao uso de aplicativos desenvolvidos para área de saúde (mHealth), o que possibilita automatizar a coleta maciça de dados, integrando informações referentes à localização geográfica dos serviços, registros de imagens, sons e outras informações que podem ser transmitidas para sistemas de cloud computing.

O emprego dessas tecnologias, principalmente as de acesso livre, tem o potencial de reduzir custos e agilizar procedimentos de coleta e análise ${ }^{3,4}$. Por serem de acesso livre, elas estão disponíveis para uso sem custos, permitindo que o seu emprego seja feito em países, estados ou municípios que possuem recursos escassos, e que, por isso, precisam alocá-los de forma eficiente. No entanto, um dos principais desafios é integrar métodos de avaliação estabelecidos na literatura com tais recursos tecnológicos de acesso livre. Tais métodos têm como potencial o auxílio na gestão dos sistemas de saúde e na abordagem a alguns de seus obstáculos, enquanto ferramentas de apoio ao trabalho, permitindo atualizações e futuras replicações ${ }^{3-5}$.

Nessa direção, técnicas e ferramentas de georreferenciamento tornam-se importantes para a visualização, compreensão e análise de dados numéricos, tanto nas distribuições espaciais de incidência de eventos relacionados ao processo saúde-doença quanto na organização geográfica dos sistemas e serviços ${ }^{6}$. Outra importante estratégia investigativa e que pode ser atrelada aos dados provenientes do georreferenciamento é a análise de redes sociais (ARS). A ARS focaliza os aspectos relacionais, ou seja, como os pontos de um todo se relacionam, possibilitando identificar e mensurar as interações existentes, bem como compreender o papel das partes dentro de um sistema ${ }^{7}$. A justificativa para sua utilização se deve ao fato de os sistemas de saúde não serem constituídos apenas pelos serviços, mas também pelas interações estabelecidas entre eles, numa perspectiva relacional ${ }^{8}$. Dessa forma, para uma compreensão mais contextualizada desses sistemas, além do levantamento dos componentes, é necessário identificar também as formas como se relacionam, quais são os dispositivos centrais e os fluxos estabelecidos.

Especificamente na abordagem assistencial à temática do uso de drogas, tais perspectivas adquirem uma relevância ainda maior. Atualmente o abuso de drogas coloca-se como um problema social e de saúde pública a ser tratado prioritariamente por políticas9. De modo a abarcar a complexidade deste problema, a oferta de cuidado aos usuários deve ser contextualizada às necessidades das pessoas, à gravidade do problema e aos riscos associados. Tal perspectiva sugere que diversos serviços e múltiplos setores estejam envolvidos e articulados, construindo uma rede articulada de serviços que potencialize o uso dos seus recursos. Dessa maneira, a oferta do cuidado envolve serviços especializados de base comunitária, variadas modalidades e diferentes abordagens de tratamento, prevenção e promoção de saúde ${ }^{10,11}$.

Nesse sentido, de acordo com Strang e colaboradores ${ }^{12}$, as diferentes formas de oferta das intervenções e organização dos serviços assistenciais na área constituem importantes fatores a serem considerados na análise das políticas públicas. Essa perspectiva é corroborada pelo fato de que mesmo tratamentos com alta eficácia produzirão poucos benefícios se forem inacessíveis e desarticulados do restante dos outros 
serviços $^{12}$. Sendo assim, compreender de que forma esses serviços se distribuem espacialmente e se organizam em sistemas e redes, torna-se um aspecto fundamental para os processos avaliativos e tomadas de decisão na área.

Tais processos avaliativos são complexos e poucos são os estudos orientados para tal tipo de análise na área de drogas. Conforme aponta Babor e colaboradores ${ }^{10}$, a maior parte das pesquisas sobre tratamento de usuários de drogas foca os aspectos clínicos ou o processo terapêutico, deixando importantes lacunas referentes à análise dos diferentes tipos e organização dos serviços.

Destarte, a proposição de métodos viáveis para análise da organização de serviços em sistemas de cuidado, especialmente na área de drogas, tem potencial de gerar impacto na avaliação, planejamento e tomada de decisões referentes à oferta de cuidado aos usuários de drogas. Iniciativas inovadoras, agregando o mapeamento dos territórios, georreferenciamento dos serviços e a sua caracterização pela ARS, a partir de ferramentais de acesso livre, podem contribuir para a minimização do cenário em que as investigações científicas e processos avaliativos desempenham um papel aquém de seu potencial na configuração dos serviços e sistemas de tratamento dos usuários de drogas em grande parte dos países ${ }^{12,13}$.

A partir do exposto anteriormente, o presente artigo visa apresentar um método de georreferenciamento e uma análise de sistemas de cuidado aos usuários de drogas baseado em tecnologias e processos de livre acesso que podem ser utilizados em diferentes cenários.

\section{Método}

Inicialmente, será descrito o delineamento geral do método e, posteriormente, como foi realizada a sua utilização em um município brasileiro de médio/grande porte, de modo a demonstrar de forma prática alguns dos resultados provenientes, bem como as suas potencialidades e a subsidiar sua aplicação em outros contextos. O município escolhido foi Juiz de Fora, no estado de Minas Gerais. Sua extensão territorial em 2016 era de aproximadamente $1.436 \mathrm{~km}^{2}$ e, em 2010, sua densidade demográfica era de 359,59 hab $/ \mathrm{km}^{2}$ e o grau de urbanização, em atingiu 99,2. A população estimada pelo IBGE era de 545.942 habitantes, com Índice de Desenvolvimento Humano de o,778 em 2010 e Produto Interno Bruto de R\$7.180.719,00 ${ }^{14}$. O sistema municipal de tratamento dos usuários de drogas foi definido conceitualmente, teve seus serviços identificados e mapeados por georreferenciamento e, posteriormente, suas relações foram caracterizadas a partir da ARS. De forma geral, o método é constituído por três etapas, que serão explicitadas abaixo.

\section{Definição conceitual dos sistemas de tratamento e redes de atenção aos usuários de drogas}

Como primeira etapa, deve-se buscar a definição conceitual do objeto de estudo. No caso dos sistemas de tratamento dos usuários de drogas, isso significa identificar: (a) quais são seus componentes (setores, níveis de atenção, serviços e outros recursos integrantes); (b) os princípios que orientam esses sistemas; e (c) como esses elementos devem se organizar e se articular, isto é, os processos relacionais. Para isso, sugere-se a fundamentação das políticas públicas e/ou dos mecanismos normativos da área, como leis e portarias, entre outros documentos e diretrizes organizativas. Cabe ressaltar que essa definição conceitual pode variar de acordo com as particularidades dos países, estados e municípios.

A partir dessa delimitação inicial do objeto de estudo, procede-se a definição de processos e ferramentas. Para o levantamento dos componentes da rede, três abordagens são propostas: (a) levantamento preliminar da rede através de ferramentas e documentos públicos da gestão municipal; (b) aprofundamento do levantamento preliminar por bases de dados públicas de órgãos ministeriais (Ministérios da Saúde e da Assistência Social e Secretaria Nacional de Políticas sobre Drogas) e contato com secretarias gestoras 
municipais e entidades de participação social sobre a temática; e (c) método bola de neve com profissionais da rede. Estas abordagens em conjunto também se encontram em conformidade para a posterior utilização da ARS em redes consideradas sociocêntricas, caracterizando-se como um survey por saturação (saturation survey)7,15. Cabe destacar que essa abordagem de mapeamento completo da rede, em formato de inquérito, é fortemente recomendada e colocada como condição necessária para compreensão dos dados relacionais sobre os serviços, visando apontar as relações existentes e as inexistentes que caracterizam serviços isolados na rede ${ }^{16}$.

\section{Caracterização da rede de atenção aos usuários de drogas no Brasil}

No caso do Brasil, os sistemas de tratamento no âmbito municipal são organizados por meio de redes de atenção aos usuários de drogas. Portanto, para compreender a caracterização dessas redes, tomou-se como base as políticas e instrumentos normativos brasileiros sobre drogas ${ }^{17-19}$. A partir da noção ampliada de rede postulada pelas políticas brasileiras, os serviços que prestam tratamento aos usuários de drogas podem ser governamentais ou não governamentais, e as modalidades de tratamento diversificadas e constituintes das seguintes esferas: 1) Saúde, conformada pelos dispositivos da Rede de Atenção Psicossocial (Raps), que integra o Sistema Único de Saúde (SUS), responsáveis pela assistência a pessoas com sofrimento ou transtorno mental e com necessidades decorrentes do uso de drogas; 2) Assistência social, através dos serviços do Sistema Único de Assistência Social (Suas) responsáveis pelas ações de promoção e proteção social; e 3) Recursos assistenciais comunitários com as instituições assistenciais oriundas da própria comunidade como forma complementar de ampliação e aprofundamento do escopo de cuidado. O Quadro 1 apresenta os componentes e as características da rede de atenção aos usuários de drogas. 
Quadro 1.- Configuração da rede de atenção aos usuários de drogas

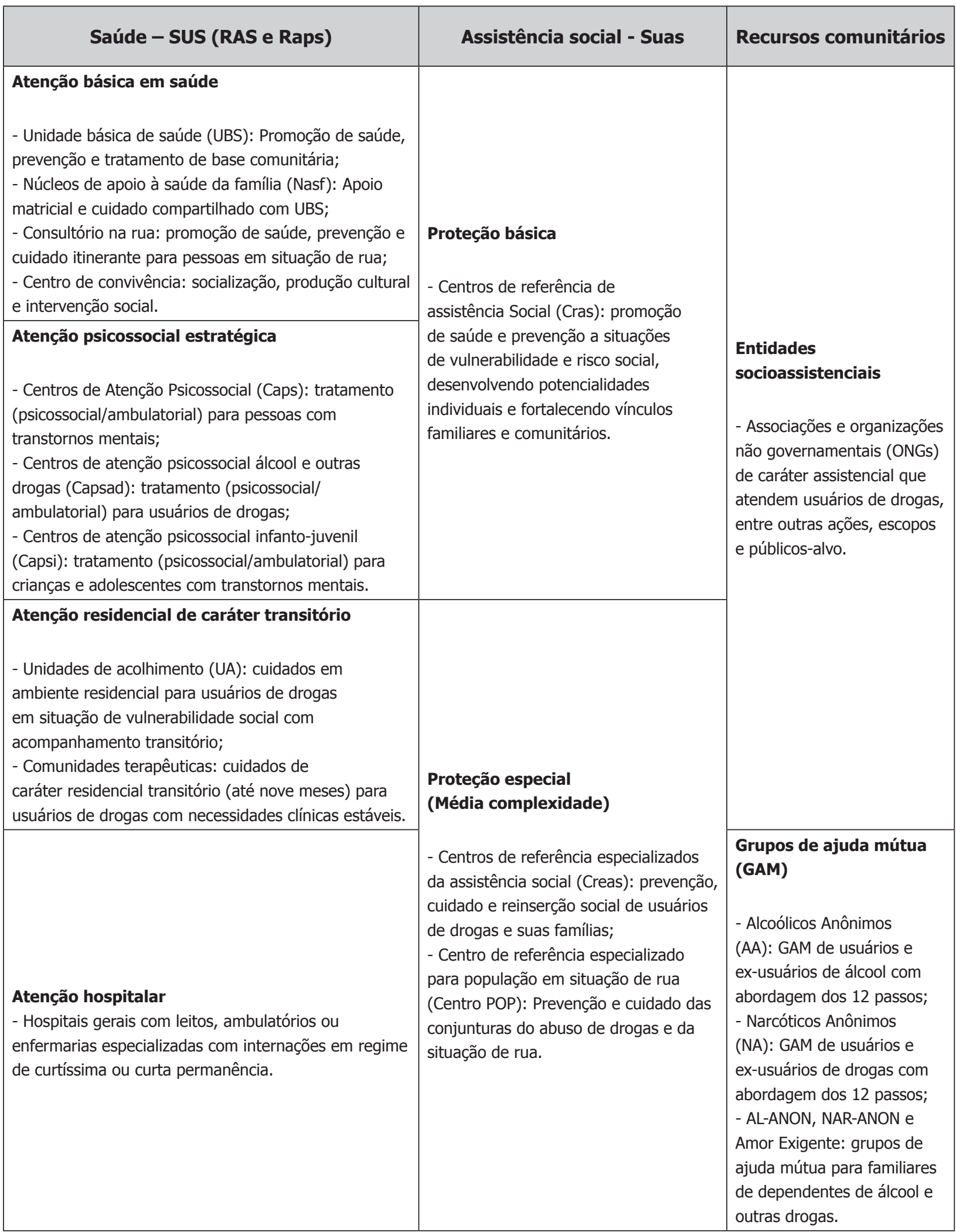


Quadro 1.- Configuração da rede de atenção aos usuários de drogas (continuação)

\begin{tabular}{|c|c|c|}
\hline Saúde - SUS (RAS e Raps) & Assistência social - Suas & Recursos comunitários \\
\hline $\begin{array}{l}\text { Atenção de urgência e emergência } \\
\text { - Serviço de Atendimento Móvel de Urgência (Samu), } \\
\text { unidades de pronto atendimento (UPA), Hospitais de } \\
\text { Pronto Socorro: classificação de risco e cuidado nas } \\
\text { situações de urgência e emergência. }\end{array}$ & \multirow{3}{*}{$\begin{array}{l}\text { Proteção Especial } \\
\text { (média complexidade) } \\
\text { - Serviços de acolhimento institucional: } \\
\text { cuidado residencial de curta e média } \\
\text { permanência para pessoas e famílias } \\
\text { afastadas temporariamente de seu } \\
\text { núcleo familiar e/ou comunitário de } \\
\text { referência. }\end{array}$} & \\
\hline $\begin{array}{l}\text { Estratégias de desinstitucionalização } \\
\text { - Serviços residenciais terapêuticos (SRTs): ambientes } \\
\text { residenciais para os usuários de drogas e pessoas com } \\
\text { transtorno mental egressos de longa permanência em } \\
\text { hospitais psiquiátricos. }\end{array}$ & & \\
\hline $\begin{array}{l}\text { Estratégias de reabilitação psicossocial } \\
\text { - Iniciativas intersetoriais de geração de trabalho e } \\
\text { renda, empreendimentos solidários e cooperativas. }\end{array}$ & & \\
\hline
\end{tabular}

Fontes: Brasil, 2004; Brasil, 2005; Brasil, 2011.

\section{Levantamento dos serviços e seu mapeamento por georreferenciamento}

No que se refere ao mapeamento por georreferenciamento dos serviços levantados, ferramentas de acesso disponíveis na internet são essenciais por apresentarem baixo custo, e até mesmo, em alguns casos, serem gratuitas e permitirem a replicação por outros pesquisadores, por atores estatais e profissionais que objetivam divulgar informações sobre saúde como recurso para tomada de decisões. Para tanto, através de buscas na internet avaliando a disponibilidade de recursos necessários e suas usabilidades, optou-se por ferramentas online disponibilizadas pelo Google Inc. Estas, além de atenderem aos critérios propostos, não exigem conhecimento de programação ou técnicas avançadas de georreferenciamento, ampliando o escopo de uso do método proposto. As ferramentas escolhidas para o levantamento foram os formulários (forms) e tabelas em planilhas eletrônicas (spreadsheets) do Google Drive (GD). Para o georreferenciamento, foram utilizadas as ferramentas de endereços do Google Fusion Tables, aplicativo também integrado com o Google Drive, além do Google Earth utilizado para obter coordenadas geográficas de serviços localizados em endereços rurais que não eram georreferenciados automaticamente pelo Google Fusion Tables. Para utilizar as ferramentas do GD (como as planilhas, formulários e o Google Fusion Tables) é necessário somente uma conta no Google e um computador ou dispositivo móvel com acesso à internet.

O georreferenciamento foi planejado a partir dos endereços dos serviços. Assim, o primeiro passo do mapeamento da rede consistiria na criação de um formulário (form) no GD, servindo como interface gráfica amigável para registro dos serviços levantados, e contendo, além de seus endereços, outras informações relevantes como o tipo de serviço, público-alvo, modelo de tratamento etc. Esses dados podem ser coletados via contato telefônico com profissionais dos serviços, sendo inseridos pelos pesquisadores no mencionado formulário e automaticamente transpostos para uma planilha (spreadsheet) do GD.

A partir dessa planilha é criada uma Fusion Table. O Google Fusion Tables é um serviço de computação em nuvem (cloud computing) online com objetivo de armazenar dados e disponibilizar informações visuais através de mapas geográficos, fluxogramas, gráficos e tabelas. Estes recursos podem ter o seu acesso compartilhado na internet por meio de links, ou ainda pela incorporação desses gráficos e mapas em páginas da internet que utilizam o formato HTML (Hypertext Markup Language)². 
Nos casos de serviços com problemas de localização e georreferenciamento, foi feito o download da Fusion Table em formato KML (Keyhole Markup Language) e importada para uma versão gratuita do programa Google Earth. A KML é um formato de arquivo usado para exibir dados geográficos ${ }^{20}$. No Google Earth foram localizadas as coordenadas geográficas (longitude e latitude) de todos os serviços e feito o georreferenciamento a partir dessas coordenadas, ao invés dos endereços. Além disso, foi acrescentada a representação administrativa do município - referente às suas unidades territoriais e regiões geográficas - através de camadas (layers) nos mapas. Essas camadas foram extraídas dos dados de malhas digitais dos setores censitários do censo de 2010 de Minas Gerais ${ }^{21}$, sendo o Google Earth utilizado para abrir os arquivos do tipo SHP (Shape File) referentes a essas malhas que armazenam em formato vetorial informações de posição, formato e atributos de feições geográficas.

Posteriormente, foi extraído do Google Earth um arquivo com os serviços levantados, suas informações e as camadas territoriais do município (novamente em formato KML) e, em seguida, importado novamente para o Google Fusion Tables. Dessa vez, para o georreferenciamento desses serviços pelo Fusion Tables, ao invés de utilizar os seus endereços, foi utilizada a relação latitude/longitude proveniente do formato de arquivo KML.

A partir desse georreferenciamento, os diferentes tipos de serviços foram representados como pontos no mapa, utilizando símbolos de identificação (marcadores) disponibilizados pelo Google (Google markers). Esses pontos foram plotados em um mapa geográfico criado no Fusion Tables que mescla imagens de satélites a informações administrativas georreferenciadas de diversos países, estados e municípios.

\section{Caracterização dos relacionamentos entre os serviços da rede a partir da ARS}

Para a etapa de caracterização dos relacionamentos entre os serviços a partir da ARS, também são indicados softwares e/ou plataformas disponibilizados gratuitamente na internet, sendo eles: ODK ${ }^{22}$, Formhub $^{23}, \mathrm{R}^{24}$ e Gephi ${ }^{25}$. Com base em estudos prévios ${ }^{7,26,27}$, a abordagem escolhida para a ARS foi a sociocêntrica, visando abarcar todos os serviços (caracterizados por nós) que fazem parte da rede e as ligações (laços) de uns com os outros ${ }^{15}$.

A adoção da metodologia de análise de redes sociais exige que cada elemento da rede avalie e seja avaliado por todos os demais elementos da mesma. Neste contexto, a possibilidade de criação de formulários eletrônicos inteligentes é especialmente importante para garantir a viabilidade da realização das entrevistas. A programação de condições lógicas que permitam aos entrevistadores saltar grande número de questões com base em respostas prévias, garante maior agilidade na aplicação. Para tanto, a utilização do padrão XLSForms na construção de questionários e instrumentos de coleta dos dados permite a criação desses formulários sem utilizar uma linguagem de programação, basta que as questões e suas condicionais sejam inseridas em uma planilha do Google Spreadsheets adotando o padrão XLSForms. Através de um arquivo do tipo XLS (Excel Spreadsheet) gerado a partir dessa planilha, a base do formulário eletrônico é inserida na plataforma online Formhub, que é gratuita e de código aberto, baseada nas ferramentas do Open Data Kit (ODK). Este possui soluções para a construção, gestão e o compartilhamento de surveys utilizando plataformas móveis off-line para coleta de dados (tablets e celulares, ainda que sem acesso à internet).

Através da integração da plataforma do Formhub com o aplicativo ODK Collect pode-se gerar uma interface gráfica no formato de um aplicativo para sistemas Android que permite a coleta dos dados, o armazenamento e o envio dos dados através de um conexão com a internet (ou via USB) para o servidor ODK Aggregate ${ }^{22}$. Atualmente esta plataforma tem sido utilizada para levantamento de dados via aparelho móvel, possibilitando inclusive o registro de localização por GPS (muitas vezes disponível nos próprios aparelhos móveis). 
Após o preenchimento das entrevistas, os dados são exportados, em forma de matrizes de adjacência, do repositório no Formhub para o software estatístico R, utilizando o pacote formhub. Posteriormente, essas matrizes são exportadas do R para o software Gephiv. 0.8.2 ${ }^{25}$ objetivando a construção de sociogramas e extração de métricas.

\section{Resultados: utilização do método em um município brasileiro de médio porte}

\section{Levantamento dos serviços componentes da rede de atenção}

O levantamento dos serviços no município iniciou-se através do contato da equipe de pesquisadores, composta por quatro pessoas, com a gestão municipal, com as secretarias gestoras, para ter acesso aos serviços públicos municipais da saúde e de assistência social existentes e aos programas que poderiam ser utilizados para o trabalho com a temática do uso de drogas. Além do material proveniente deste contato, utilizou-se como base um catálogo feito pela Secretaria Municipal de Assistência Social contendo entidades socioassistenciais do município. Nesse levantamento preliminar, todo realizado por telefone, foram identificados 121 serviços que prestavam algum tipo de assistência a usuários de drogas.

Esse levantamento inicial foi aprofundado a partir da busca de informações via internet nas bases ministeriais supracitadas e por meio de contato por telefone com o Conselho Municipal de Assistência Social e com o Conselho Municipal de Políticas Integradas sobre Drogas. A partir desse aprofundamento, foi possível identificar 46 novos serviços que prestavam assistência a usuários de drogas no município, totalizando, até então, 167 dispositivos.

Seguindo o processo do levantamento por saturação, a partir da obtenção desses dados utilizou-se o método de amostragem bola de neve (snowball) ${ }^{28}$. Inicialmente, foram identificados atores-chave da rede, que eram profissionais dos serviços. Estes foram entrevistados para que pudessem sinalizar a existência de outros serviços que não constavam no levantamento inicial. Nos casos de surgimento de novas instituições, profissionais desses serviços foram entrevistados para a identificação de novos dispositivos até que fossem repetidos todos os serviços já identificados. No final dessa etapa, sete serviços foram excluídos, por não mais estarem em funcionamento, e 27 serviços até então não identificados foram incluídos, resultando em 187 serviços que compuseram a rede de atenção aos usuários de drogas do município. Esses serviços são apresentados na Tabela 1. 
Tabela 1. Tipologia dos serviços que prestam assistência a usuários de drogas em Juiz de Fora

\begin{tabular}{lcc}
\hline Tipos de serviço & $\mathbf{N}$ & $\mathbf{\%}$ \\
Unidades básicas de saúde (UBS) urbanas & 48 & 26,1 \\
Unidades básicas de saúde (UBS) rurais & 19 & 10,4 \\
Centros de atenção psicossocial (Caps) gerais & 3 & 1,6 \\
Centro de Atenção Psicossocial - Álcool e Drogas (Capsad) & 1 & 0,5 \\
Centro de Atenção Psicossocial infantil (Capsi) & 1 & 0,5 \\
Ambulatórios de saúde mental, álcool e outras drogas & 2 & 1,1 \\
Hospitais gerais com leitos, ambulatórios ou enfermarias para & 5 & 3,3 \\
álcool e outras drogas & & \\
Serviço de Atendimento Móvel de Urgência (Samu) & 1 & 0,5 \\
Centros de referência de assistência social (Cras) & 9 & 4,9 \\
Centros de referência especializados de assistência social (Creas) & 4 & 2,3 \\
Equipe de consultório na rua & 2 & 0,5 \\
Serviços residenciais terapêuticos (SRT) & 12 & 6,5 \\
Serviços de acolhimento institucional para adultos & 3 & 1,6 \\
Centros de convivência & 1 & 0,5 \\
Entidades socioassistenciais & 10 & 4,9 \\
Clínicas e comunidades terapêuticas & 19 & 10,9 \\
Grupos de ajuda mútua & 47 & 23,9 \\
Total & $\mathbf{1 8 7}$ & $\mathbf{1 0 0 , 0}$ \\
\hline
\end{tabular}

Fonte: Elaborada pelos autores

De modo geral, observa-se a importância dos níveis de atenção e dispositivos de caráter generalista no fomento a perspectivas de cuidado abrangentes e de cunho territorializado e comunitário, como a atenção básica, as UBS na saúde, a proteção básica e os Cras na assistência social, concebidos numa perspectiva intersetorial. Ademais, no que se refere aos dispositivos especializados, é destacada a proeminência numérica dos dispositivos não governamentais, como os GAM e as comunidades terapêuticas, em detrimento dos dispositivos públicos que possuem essa natureza e intencionalidade, como, por exemplo, os Caps e o Capsad.

\section{Mapeamento dos serviços da rede por georreferenciamento}

A partir do método supracitado, obtivemos como principal resultado o georreferenciamento de todos os 187 serviços levantados da rede de atenção aos usuários de drogas de Juiz de Fora, assinalando suas respectivas disposições pelo território da cidade. Estes serviços e sua distribuição foram incorporados em uma mapa juntamente com links em cada marcador referente aos serviços contendo as informações coletadas durante o levantamento (Figura 1). Todo o processo de levantamento e mapeamento está representado na Figura 2. A rede georreferenciada foi disponibilizada gratuitamente em uma página da internet, com os serviços existentes marcados em suas respectivas localidades, junto de informações, como tirar suas tipologias, modelos de tratamento, público-alvo e telefone. 


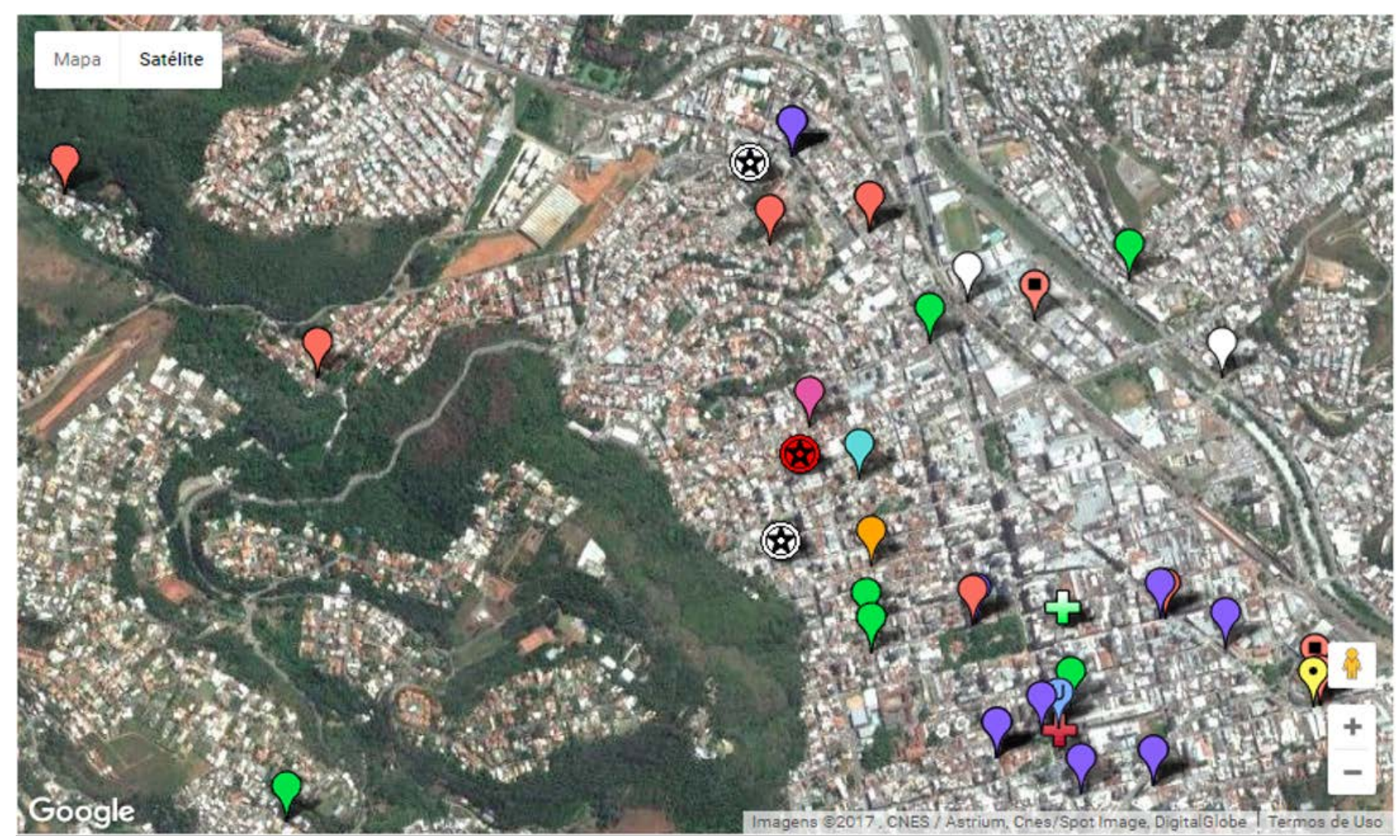

Legendas

(8) Centro de Atenção Psicossocial geral (CAPS)

Centro de Atenção Psicossocial - Álcool e Drogas (CAPSad)

$\nabla$ Entidades Socioassistenciais

$\checkmark$ Clínicas e Comunidades Terapêuticas

$\checkmark$ Grupos de Ajuda Mútua

Serviços de Acolhimento Institucional para adultos

$\nabla$ Centro de Convivência

$\curlyvee$ Serviços Residenciais Terapêuticos (SRT)

Serviço de Atendimento Móvel de Urgência (SAMU)

C Centros de Referência da Assistência Social (CRAS)

$\nabla$ Centros de Referência Especializados da Assistência Social (CREAS)

(4) Unidades Básicas (UBS) urbanas

A Ambulatórios em saúde mental e álcool e outras drogas

की Hospitais gerais com leitos, ambulatórios ou enfermarias para álcool e outras drogas

Figura 1. Mapa com a distribuição dos serviços

Fonte: Google Fusion Tables 


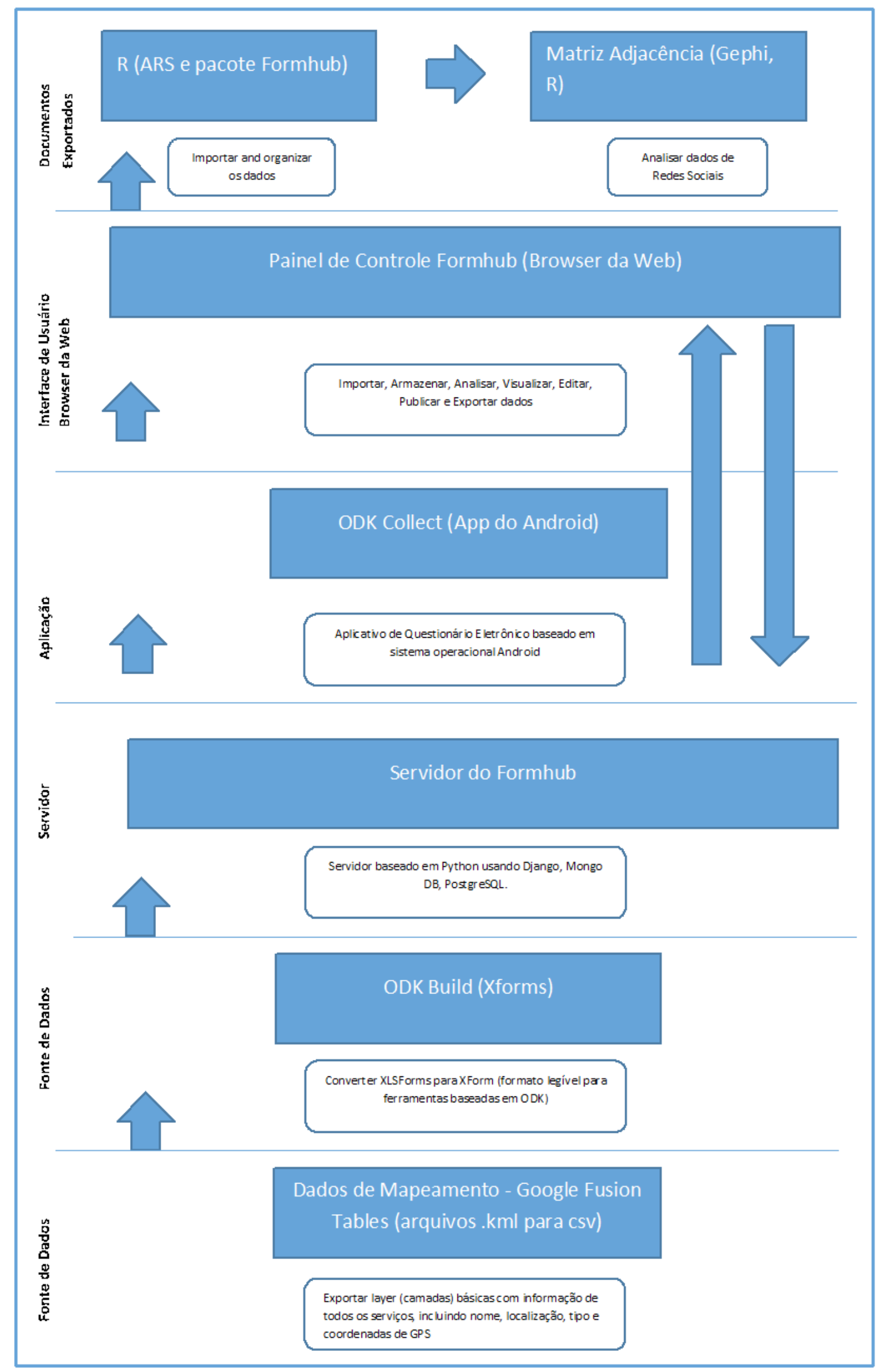

Figura 2. Fluxograma do mapeamento por georreferenciamento da rede Fonte: Elaborada pelos autores 


\section{Caracterização dos relacionamentos entre os serviços a partir da análise de redes sociais}

Os dados referentes aos relacionamentos foram coletados com questionários aplicados por pesquisadores previamente treinados, na forma de entrevistas estruturadas, individuais e em grupos, de acordo com as possibilidades dos participantes que eram profissionais dos serviços descritos. Para a escolha desses profissionais, os quatro pesquisadores contataram todos os coordenadores/gestores dos serviços e perguntaram a eles quem eram os profissionais com maior conhecimento sobre a rede de atenção aos usuários de drogas.

Com os instrumentos utilizados procuramos obter respostas dos profissionais referentes às relações existentes entre os seus serviços e outros dispositivos da rede. Visando à adequação dos instrumentos, ao conteúdo, à linguagem e à forma de aplicação, foram realizados 15 pré-testes. Nesses pré-testes, feitos com profissionais de serviços semelhantes aos da pesquisa, modificamos os cabeçalhos de algumas perguntas e as formas de perguntá-las.

Como suporte para as respostas, foi confeccionado e entregue a cada respondente um cardápio/ menu com os nomes dos serviços da rede de atenção mapeados, para que pudessem consultar durante a entrevista, minimizando esquecimentos. A pergunta inicial era a seguinte: No último ano, com quais dispositivos (listados no menu) o seu serviço teve algum tipo de relação/atividade em conjunto referente à atenção aos usuários de drogas? Posteriormente, eles foram perguntados - para responderem com 'sim' ou 'não' - se os serviços em que trabalhavam mantinham outros tipos de relação com os dispositivos que mencionaram anteriormente, tais como: referência de pacientes, contrarreferência, coordenação conjunta de casos, programas em conjunto e treinamento.

Dessa forma, as relações entre os 187 serviços foram caracterizadas, possibilitando uma melhor visualização sobre como os dispositivos se articulam na provisão do cuidado aos usuários de drogas e, consequentemente, quais são as formas de estruturação dessa rede de atenção. A Figura 3 representa o sociograma da rede.

Como o escopo do presente artigo é a apresentação do método, não serão apresentados resultados detalhados provenientes da utilização da ARS na compreensão do sistema e rede de atenção aos usuários de drogas abarcado. Entretanto, cabe ressaltar que as medidas utilizadas pela ARS são diversas e aplicadas de acordo com o propósito do estudo e as características da rede que se pretende analisar. Sendo assim, podem ser selecionadas métricas de: a) centralidade, que dizem respeito à posição e ao papel exercido pelos 'nós' dentro da rede e o quanto essa posição influencia outros 'nós' e o fluxo da rede; identificam os serviços mais proeminentes, com um grande número de relações dentro da rede, isto é, dispositivos centrais; b) coesão, referentes à estrutura geral da rede, como densidade, distância entre os 'nós', transitividade, existência de clusters etc. ${ }^{716}$. Para um maior contato com os achados provenientes da ARS na avaliação dos sistemas e redes de cuidado aos usuários de drogas, ver Costa e colaboradores ${ }^{29}$. 


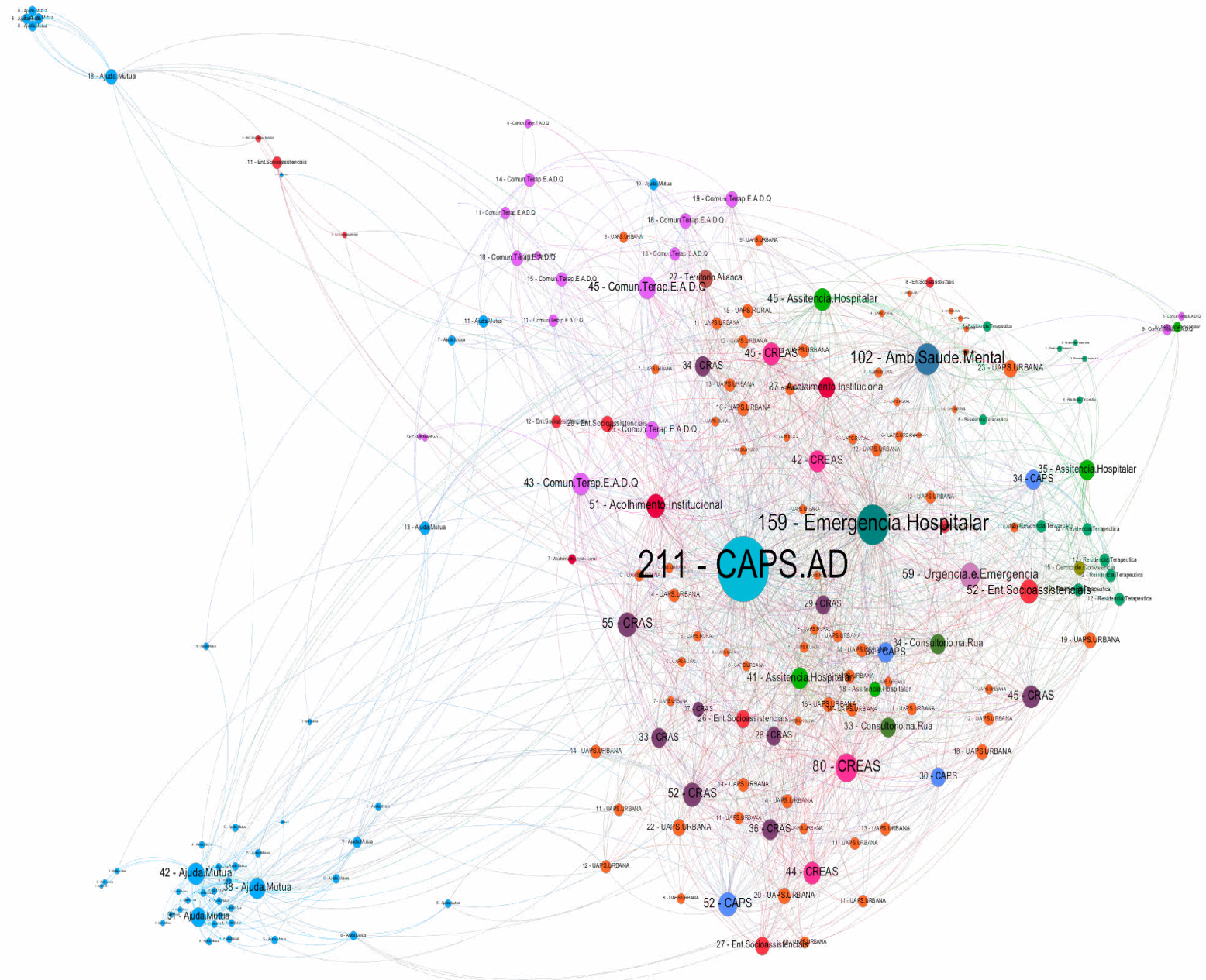

Figura 3. Sociograma da rede Fonte: Elaborada pelos autores

\section{Discussão}

O método apresentado possui potencial de inovação não só para os níveis de pesquisa, gestão, e atuação profissional, mas também para os usuários da rede. No que diz respeito aos pesquisadores, é possível impactar o olhar comum das pesquisas epidemiológicas voltadas para atributos populacionais, características dos serviços isolados ou aspectos clínicos, modificando-o em direção a uma perspectiva macro, de rede, abordando aspectos relacionais ${ }^{8,10}$. Conforme elucida Denis, "na sociedade contemporânea as políticas públicas, os programas, as decisões, são postas em funcionamento em redes”30, de modo que as avaliações relacionadas à administração pública abranjam tais características.

Para os gestores, o método pode facilitar o gerenciamento da rede, a partir do momento em que permite uma maior visualização dos serviços existentes, levantando pontos de insuficiência que precisam ser minimizados ou revertidos, e pontos de facilitação, a serem potencializados. Ilustrativamente, a partir da utilização do presente método, é possível observar a insuficiência de determinados dispositivos e níveis de atenção, em especial dos Capsad, de acordo com a sua quantidade e com as suas disposições territoriais e devido ao fato de serem os principais dispositivos especializados para abordar pessoas com problemas decorrentes do uso de drogas. De acordo com Gordon e Womersley ${ }^{31}$, o entendimento de um conjunto é facilitado ao ser visualizado graficamente, sendo mais fácil compreender uma rede a partir de mapas do que através de descrições ou tabelas. Para isso, estudos subsequentes podem aliar o georreferenciamento e a caracterização da rede com o levantamento de outros dados censitários, sociais e/ou territoriais dos 
municípios e regiões. Ademais, em consonância com Luger, Carrier e Power ${ }^{32}$, o georreferenciamento dos serviços possibilita a reconfiguração dos recursos de acordo com as necessidades locais e o monitoramento das mudanças ao longo do tempo.

A utilização de ferramentas de acesso livre por meio da internet, também facilita a replicação do método, inclusive, para outras áreas. Não são demandadas equipes de recursos humanos extensas, nem custos adicionais aos já empregados na constituição da infraestrutura de laboratórios de pesquisas e secretarias, como a existência de telefones, computadores e provedores de internet, permitindo um pequeno investimento de tempo e deslocamento somente para as entrevistas. Por utilizar tecnologias de fácil acesso, o método proposto também pode ser constantemente atualizado para comportar as mudanças ocorrentes na rede, que é dinâmica.

Devido às possibilidades do Google Fusion Tables, tanto de interpretação pelos recursos visuais de acesso livre quanto de armazenamento de grande volume de dados, esta ferramenta aparece como opção interessante, pelo fato de não exigir conhecimentos específicos de linguagem de programação. Num primeiro momento, alguns serviços não foram reconhecidos geograficamente (principalmente aqueles localizados em zonas rurais). Contudo, a utilização conjunta do Google Fusion Tables e do Google Earth, provendo dados espaciais de latitude e longitude, suplantou este empecilho. Ademais, por ser uma forma de computação nas nuvens, o Google Fusion Tables facilita o compartilhamento de documentos e o trabalho em equipe, uma vez que não são necessários servidores específicos, instalação de softwares e utilização de memória do computador ${ }^{2}$.

No que tange às possibilidades oriundas do Formhub e ODK, salientamos que tais ferramentas são especialmente adaptáveis ao trabalho em regiões sem acesso à internet e com poucos recursos. Grande parte das experiências de sua implementação foi feita em países em desenvolvimento, em regiões que contavam com pouca estrutura no que diz respeito a tecnologias da informação e comunicação ${ }^{22}$.

A partir dos fatores supracitados, justifica-se a relevância do método apresentado, com potencial de incorporação e replicação por outras áreas, setores e instituições brasileiras e ainda em contextos internacionais. A fim de melhor entender e esclarecer a sua complexidade, as análises de sistemas abrangentes e, consequentemente, das redes de atenção aos usuários de drogas, devem primeiramente identificar seus componentes. Contudo, na maioria das vezes, as pesquisas na área param nesse ponto, entendendo esses sistemas e redes como uma mera junção de serviços, numa perspectiva denominada por Frenk ${ }^{8}$ como abordagem de inventário (inventory approach), ou seja, um mostruário/cardápio de serviços.

Os sistemas e a rede de atenção aos usuários de drogas devem ser concebidos para além da identificação dos serviços existentes e aspectos meramente estruturais, por meio de uma abordagem relacional (relational approach $)^{8}$, uma vez que envolvem pessoas e são influenciados pelas interações estabelecidas e por diferentes aspectos, como modelos de cuidado, concepções/valores etc. Em consonância com Babor e colaboradores ${ }^{10}$, entende-se, que os sistemas e redes de cuidado não são somente conjuntos de serviços e recursos. Neste sentido, a análise de redes sociais aparece como uma ferramenta metodológica importante na compreensão desses sistemas e redes de atenção aos usuários de drogas, analisando interações e aspectos relacionais de serviços, profissionais e também usuários, identificando dispositivos centrais, fluxos assistenciais etc.7,27

Limitações do presente estudo que podem ser minimizadas em pesquisas subsequentes referem-se à utilização de métodos de amostragem baseados em nominação sucessiva que sejam submetidos a verificações e ajustes posteriores, permitindo um maior controle sobre os resultados de sua aplicação. Futuras pesquisas e modelos estatísticos, a partir da respondent-driven sampling (RDS), comumente utilizada em populações difíceis de se contatar (hard-to-reach-populations), podem contribuir na minimização dessas limitações ${ }^{33,34}$. 


\section{Considerações finais}

O presente artigo visou apresentar um método de georreferenciamento e de análise de sistemas e redes de cuidado aos usuários de drogas baseado em tecnologias e processos de acesso livre, para que possam ser replicados em diferentes cenários, abarcando variadas temáticas. A partir de sua utilização num contexto específico e dos resultados obtidos, considera-se que o presente método descrito e discutido, juntamente com as tecnologias que o constituem, permitem que seja empregado, agregando potencialidades para o trabalho na gestão, assim como para a atuação profissional e a pesquisa com os sistemas e redes de atenção ao usuários de drogas.

Em suma, merecem destaque as seguintes potencialidades: 1) o caráter ampliado proporcionado pela abordagem de redes; 2) o baixo custo e a utilização de ferramentas inovadoras e acessíveis; 3) o auxílio para a visualização e o gerenciamento da rede. A partir do presente trabalho, abrem-se possibilidades não só para a utilização do método aqui apresentado, mas também para clarificações contextualizadas e discussões mais bem fundamentadas acerca das variadas configurações dos sistemas e redes assistenciais aos usuários de drogas existentes no Brasil.

\section{Referências}

1. Gibbons MC. eHealth solutions for healthcare disparity. New York: Springer; 2008.

2. Gonzalez H, Halevy A, Jensen CS, Langen A, Madhavan J, Shapley R, Shen W. Google fusion tables: data management, integration and collaboration in the cloud. Proceedings of the ACM Symposium on Cloud Computing. New York: ACM; 2010:175-80.

3. Källander K, Tibenderana JK, Akpogheneta OJ, Strachan DL, Hill Z, ten Asbroek AH et al. Mobile health (mHealth) approaches and lessons for increased performance and retention of community health workers in low- and middle-income countries: a review. J Med Internet Res [Internet]. 2013 [citado em 2017 mar. 23]; 15(1):e17. Disponível em: https://www.ncbi.nlm.nih.gov/pmc/articles/PMC3636306/

4. Labrique $A B$, Vasudevan $L$, Kochi E, Fabricant R, Mehl G. mHealth innovations as health system strengthening tools: 12 common applications and a visual framework. Glob Health Sci Pract. [Internet]. 2013 [citado em 2017 mar. 23]; 1(2):160-71. Disponível em: http://www.ghspjournal.org/ content/1/2/160.full

5. Pavluck A, Chu B, Mann Flueckiger R, Ottesen E. Electronic Data Capture Tools for Global Health Programs: Evolution of LINKS, an Android-, Web-Based System. Plos Negl Trop Dis. [Internet]. 2014 [citado em 2017 mar. 23]; 8(4):e2654. Disponível em: http://journals.plos.org/plosntds/ article?id=10.1371/journal.pntd.0002654

6. Cavicchio Neto V, Chiari NS, Carvalho I, Pisa IT, Alves D. Developing and Integration of Georeferencing Dynamic maps to Health Surveillance. J Health Inform. [Internet]. 2014 [citado em 2017 mar. 23]; 6(1):3-9. Disponível em: http://www.jhi-sbis.saude.ws/ojs-jhi/index.php/jhi-sbis/article/view/284/185

7. Blanchet $\mathrm{K}$, James $\mathrm{P}$. How to do (or not to do) a social network analysis in health systems research. Health Policy Plan. [Internet]. 2011 [citado em 2017 mar. 23]; 27(5):438-46. Disponível em: https:// www.ncbi.nlm.nih.gov/pubmed/21840934.

8. Frenk J. Dimensions of health system reform. Health Policy [Internet]. 1994 [citado em 2017 mar. 23]; 27(11):19-34. Disponível em: https://www.ncbi.nlm.nih.gov/pubmed/10133134

9. United Nations Office on Drugs and Crime. World Drug Report 2015. Viena: UNODC; 2015.

10. Babor TF, Stenius K, Romelsjo A. Alcohol and drug treatment systems in public health perspective: mediators and moderators of population effects. Int J Methods Psychiatr Res. [Internet]. 2008 [citado em 2017 mar. 23]; 17(S1):S50-9. Disponível em: https://www.ncbi.nlm.nih.gov/pubmed/18543363

11. Rush B. Tiered frameworks for planning substance use service delivery systems: origins and key principles. Nordic Studies on Alcohol and Drugs [Internet]. 2010 [citado em 2017 mar. 23]; 27:617-636. Disponível em: http://www.nordicwelfare.org/PageFiles/4930/08 Rush.pdf 
12. Strang J, Babor TF, Caulkins JP, Fischer B, Foxcroft DR, Humphreys K. Drug policy and the public good: evidence for effective interventions. Lancet [Internet]. 2012 [citado em 2017 mar. 23]; 379(9810):7183. Disponível em: http://thelancet.com/journals/lancet/article/PIIS0140-6736(11)61674-7/abstract

13. Rush B. The evaluation of treatment services and systems for substance use disorders. Rev psiquiatr Rio Gd Sul [Internet]. 2003 [citado em 2017 mar. 23]; 25(3):393-411. Disponível em: http://www.scielo.br/ scielo.php?script $=$ sci arttext $\&$ pid $=$ S0101-81082003000300002

14. IBGE. [Internet]. Cidades@; 2014. - [citado em 2017 mar. 23]. Disponível em: http://cidades.ibge.gov. br/painel/painel.php?codmun $=313670$

15. Smith KP, Christakis NA. Social Networks and Health. Annu Rev Sociol [Internet]. 2008 [citado em 2017 mar. 23]; 34:405-29. Disponível em: http://www.annualreviews.org/doi/abs/10.1146/annurev. soc.34.040507.134601

16. Hawe $\mathrm{P}$, Webster $\mathrm{C}$, Shiell A. A glossary of terms for navigating the field of social network analysis. J Epidemiol Community Health [Internet]. 2004 [citado em 2017 mar. 23]; 58:971-5. Disponível em: https://www.ncbi.nlm.nih.gov/pmc/articles/PMC1732636/

17. Brasil. Ministério da Saúde. A política do Ministério da Saúde para atenção integral a usuários de álcool e outras drogas. Brasília (DF); 2004.

18. Brasil. Secretaria Nacional Antidrogas. Política Nacional sobre Drogas. Brasília (DF); 2005.

19. Brasil. Portaria no 3.088 de 23 de dezembro de 2011. Institui a Rede de Atenção Psicossocial para pessoas com sofrimento ou transtorno mental e com necessidades decorrentes do uso de crack, álcool e outras drogas, no âmbito do Diário Oficial da União. Brasília (DF); 2011.

20. OGC, OGC KML Standard. 2008. [citado em 2014 fev 20]. Disponível em: http://www.opengeospatial. org/standards/kml

21. IBGE. [Internet]. [citado em 2014 fev 20]. Disponível em: http://downloads.ibge.gov.br/downloads geociencias.htm

22. Brunette W, Sundt M, Dell N, Chaudhri R, Breit N, Borriello G. Open data kit 2.0: expanding and refining information services for developing regions. Proceedings of the 14th Workshop on Mobile Computing Systems and Applications. New York: ACM; 2013:10.

23. Formhub. [Internet]. New York: Columbia University, Modi Research Group; 2012. [citado em 2017 mar. 23]. Disponível em: http://formhub.org/

24. R Development Core Team. R: A language and environment for statistical computing. Vienna: R Foundation for Statistical Computing; 2011.

25. Bastian M, Heymann S, Jacomy M. Gephi: an open source software for exploring and manipulating networks. International AAAI Conference on Weblogs and Social Media. San Jose: AAAI Publications; 2009.

26. Bittencourt ONS, Neto FJK. Rede Social no Sistema de Saúde: um Estudo das Relações Interorganizacionais em Unidades de Serviços de HIV/AIDS. Rev Adm Contemp [Internet]. 2009 [citado em 2017 mar. 23]; 13(6):87-104. Disponível em: http://www.scielo.br/scielo.php?script=sci arttext\&pid $=\mathrm{S} 1415-65552009000500007$

27. Nicaise P, Tulloch S, Dubois V, Matanov A, Priebe S, Lorant V. Using Social Network Analysis for Assessing Mental Health and Social Services Inter-Organisational Collaboration: Findings in Deprived Areas in Brussels and London. AdmPolicyMent Health [Internet]. 2013 [citado em 23 mar. 2017]; 40:331-9. Disponível em: https://www.ncbi.nlm.nih.gov/pubmed/22543978

28. Biernacki $P$, Waldford D. Snowball sampling: problems and techniques of chain referral sampling. Sociol Methods Res. [Internet]. 1981 [citado em 2017 mar. 23]; 2:141-63. Disponível em: http://journals. sagepub.com/doi/abs/10.1177/004912418101000205

29. Costa PHA, Martins LF, Medeiros AX, Salgado JA, Silva WMD, Ronzani TM et al. Sistema de referência e de contrarreferência na rede de atenção aos usuários de drogas: contribuições da análise de redes sociais. Cad Saúde Colet [Internet]. 2015 [citado em 2017 jun. 28]; 23(3):245-52. Disponível em: http://www.scielo.br/scielo.php?script=sci arttext\&pid=S1414-62X2015000300245\&lng=en

30. Denis JL. Institucionalização da avaliação na administração pública. Rev Bras Saude Mater Infant [Internet]. 2010 [citado em 2017 mar. 23] 10(Suppl.1):s229-s333. Disponível em: http://www.scielo.br/ scielo.php?script $=$ sci arttext $\&$ pid $=$ S1519-38292010000500020 
31. Gordon A, Womersley J. The use of mapping in public health and planning health services. J Public Health Med [Internet]. 1997 [citado em 2017 mar. 23]; 19(2):139-47. Disponível em: https://www.ncbi. nlm.nih.gov/pubmed/9243428

32. Luger L, Carrier J, Power R. Mapping as a method for analysing policy response in the management of health services. Health Serv Manage Res [Internet]. 2001 [citado em 2017 mar. 23]; 14:220-8. Disponível em: https://www.ncbi.nlm.nih.gov/pubmed/11725589

33. Gile KJ, Johnston LG, Salganik MJ. Diagnostics for respondent-driven sampling. Journal of the Royal Statistical Society: Series A (Statistics in Society); 2014.

34. Young AM, Rudolph AE, Quillen D, Havens JR. Spatial, temporal and relational patterns in respondentdriven sampling: evidence from a social network study of rural drug users. J Epidemiol Community Health [Internet]. 2014 [citado em 2017 mar. 23]; 68:792-8. Disponível em: https://www.ncbi.nlm.nih. gov/pubmed/24692631 